\title{
Is there a new cutoff value for high sensitivity troponin to better risk stratify patients with non-ST elevated myocardial Infarction?
}

Omar. Al-assaf ( $\square$ alassaf_94@hotmail.com )

Dubai Health Authority https://orcid.org/0000-0002-3609-4622

Anas M. Abbas

Dubai Health Authority

Hind H. Alkazim

Dubai Health Authority

Sam Codampallil Benny

Dubai Health Authority

Azan Salem BinBrek

Dubai Health Authority

Research article

Keywords: Non-obstructive myocardial infarction, Cardiac biomarkers, Coronary Angiography, Non-ST elevated myocardial infarction

Posted Date: October 19th, 2020

DOl: https://doi.org/10.21203/rs.3.rs-42900/v2

License: (c) (1) This work is licensed under a Creative Commons Attribution 4.0 International License. Read Full License 


\section{Abstract}

Background: The diagnosis of Non-ST Elevated Myocardial Infarction (NSTEMI) is dependent on elevation of high sensitivity troponin (Hs-troponin). The current cutoff point for Hs-troponin is highly sensitive but not specific for obstructive coronary artery disease (CAD). This study aims to determine the best cutoff point for diagnosing CAD in patients presented with NSTEMI.

Methods:Our study included all patients admitted as NSTEMI that underwent coronary angiography (CAG). They were grouped into two groups; A and B. Group A has obstructive CAD of $70 \%$ or more stenosis and group B with non-obstructive CAD. Patients were assessed for their demographics, clinical history, laboratory and imaging results. Using SPSS version 22, the pooled cohort of patients were analyzed at significant level $<0.05$ and the data were tested for significant correlations between two predetermined groups.

Results: Group A comprised $87.6 \%$ of the patients and both groups had a median age of 53 years. In Group A, 91\% were males, 54\% diabetics, 54\% hypertensives, and median Hs-troponin was $145 \mathrm{ng} / \mathrm{L}$. While in group B, $88 \%$ were males, $39 \%$ diabetics, $60 \%$ hypertensives, and median Hs-troponin was 54 $\mathrm{ng} / \mathrm{L}$. There was significant correlation between the two groups in the percentage of diabetes and median troponin level $(p<0.05)$. A ROC curve has identified a level of $127 \mathrm{ng} / \mathrm{dL}$ as the best cutoff of Hs-troponin in detecting obstructive CAD ( $p=0.03)$. Interestingly, $60 \%$ of patients in group $B$ had alternative diagnoses.

Conclusion:Hs-troponin is sensitive but less specific for obstructive CAD. However; increasing its cutoff value will improve its specificity.

\section{Background}

Acute coronary syndrome with normal or near normal coronary arteries, commonly known as Myocardial Infarction/Injury with Non-obstructive Coronary Arteries (MINOCA), can be caused by many etiologies like coronary disorders, myocardial disorders, or non-cardiac disorders[1-3]. These etiologies include; coronary spasm, microvascular dysfunction, myocarditis, hypertensive heart disease, stroke, sepsis, and pulmonary embolism[1,4].All these conditions result in rise of cardiac enzymes; thus, the challenge arises in making a decision whether the patient is suffering myocardial injury, ischemic infarction requiring further cardiac intervention[1].These challenging situations have increased currently with the introduction of high sensitivity cardiac troponin assay in view of its low specificity [1]. In a review article by Pasupathy et al. the prevalence of MINOCA was reported as $6 \%$, ranging from $1-15 \%$. Moreover, $14 \%$ of patients with acute myocardial infarction have been found to have non-obstructive coronary artery disease defined as coronary stenosis of less than $50 \%$. Research has shown that two third of patient with MINOCA present as NSTEMI [1,3].

Risk stratification in patients with NSTEMI is of extreme importance as VIRGO study found the prevalence of MINOCA is five times more in women than men and higher in patients with lower traditional cardiac risk factors. Another study showed that the prevalence of MINOCA is higher in younger patientsas 
well [5-8]. This has resulted in multiple risk scores being developed such as: Thrombolysis in Myocardial Infarction score, HEART score and Global Registry of Acute Coronary Events. These scores incorporate Hs-troponin as inherent factor included in the assessment of cardiovascular risks in addition to other clinical risk factors of coronary artery disease [4].All these risk scores use the current cutoff value of Hstroponin of $14 \mathrm{ng} / \mathrm{dL}$ which is sensitive but lack specificity for myocardial infarction. The American College of Cardiology recently published an article suggesting that an absolute value of Hs-Troponin of $52 \mathrm{ng} / \mathrm{dL}$ is an indictive to rule in acute myocardial injury [9]. Furthermore, the European Society of Cardiology recent 2020 guidelines have incorporated four confounders that result in rise of Hs-troponin; renal dysfunction, time to chest pain, age and gender [10].

The aim of this research is to better risk stratify patients by determining statically if a new cutoff value of Hs-troponin is of more significance than the current value of $14 \mathrm{ng} / \mathrm{dL}$ in identifying obstructive coronary artery disease in patients with NSTEMI.

\section{Methods}

This is a cohort of patients admitted between 1st of April 2018 through 31st of March 2019 in Rashid hospital. It included patients who were above 18 years old, having documented diagnosis of NSTEMI, and underwent coronary angiography regardless of the result and management strategy. The sample size was calculated at $80 \%$ power with marginal error of $5 \%$. According to the literature there is a $15 \%$ possibility of normal CAG in patients undergoing coronary angiography this will equate to a sample size of 15 patients per arm for paired $T$ test comparing the two group means [1-3]. Data was collected using a structured questionnaire present in an excel spreadsheet that included: demographics (age, gender and ethnicity), previous risk factors for cardiac disease, laboratory findings (Low Density Lipoprotein value, Hemoglobin-A1C, and highest Hs-troponin value), and CAG finding. Patients were divided into two groups according to the CAG findings. Group A with obstructive CAD defined as coronary artery stenosis of $70 \%$ or more underwent coronary intervention, or advised for surgical or medical management. And Group $B$ with non-obstructiveCAD defined as normal or non-obstructive coronary artery disease of less than $50 \%$.

Data was analyzed using SPSS software version 22. Statistical tests were carried out at $95 \%$ confidence interval and results considered significant at a p-value of 0.05 or less. For variables where their numerical data is non-skewed the mean is reported (Hemoglobin and LDL) while for variables with skewed data the median is reported (all other variables with numerical data). On the other hand, for categorical variables the percentage is reported. Correlation was investigated using T-test for non-skewed numerical data, Mann-Whitney test for skewed numerical data and chi-square test for categorical data.

An ethics approval was obtained from Dubai Scientific Research Ethics Committee, DHA holding the reference number of DSREC/RRP/2019/13.

\section{Results}


Two hundred and two patients fulfilled the inclusion criteria out of all 263 patients admitted as NSTEMI ( Table. 1 ) in the study period. Group A included 177 patients (87.6\%) of which $91 \%$ were males, meanwhile Group B had 25 patients (12.4\%) of which $88 \%$ were males. The median age in both groups was 53 years old. There is significant difference between group $A$ and $B$ in percentage of diabetics $(P=$ 0.014 ) and Median Hs-troponin $(P=0.03)$, but no significant differences reported in relation to hypertension, smoking, HbA1C, or LDL (Table.2). The confounding variables were presented in Table.3 that showed no statistical significance between both groups.

\section{Troponin as a diagnostic biomarker for myocardial infarction}

A receiver operating characteristic curve $(\mathrm{ROC})$ was obtained to statistically measure a diagnostic cutoff value for troponin as a cardiac biomarker for NSTEMI (Figure.1) considering CAG as the gold standard test. The ROC analysis showed an Area Under the Curve (AUC) of 0.634 (0.51-0.751) with a p value of 0.03 at confidence interval of $95 \%$. Studying the sensitivities and specificities of the ROC curve; it was deduced that the best cutoff value for the Hs-troponin is $127 \mathrm{ng} / \mathrm{L}$.

\section{Discussion}

Troponin has been acknowledged as a cardiac biomarker of choice for diagnosis and management of NSTEMI by the American Heart Association (AHA), American College of Cardiology (ACC) and the European Society of Cardiology $[11,12]$. However, the ACC and AHA emphasize that elevation of troponin is not always due to atherosclerotic coronary artery disease [11]. Currently, $14 \mathrm{ng} / \mathrm{L}$ is considered as the cutoff value for a positive Hs-troponin result when evaluating patients with NSTEMI. This value is highly sensitive but lack specificity. When conducting a ROC curve on our data a Hs-troponin value of $14 \mathrm{ng} / \mathrm{L}$ had a sensitivity of $99.9 \%$ with an only $4 \%$ specificity. Further studying the ROC curve, a level of $127 \mathrm{ng} / \mathrm{L}$ was the best cutoff for Hs-troponin not compromising specificity, which showed a sensitivity of $58 \%$ and specificity of $68 \%$. The test specificity will increase with increasing Hs-troponin level, while sensitivity decreases and vice versa. This curve has an AUC of 0.634 indicating a low diagnostic accuracy $(<0.7)$ despite being statistically significant $(P=0.03)$ [13]. This directs that Hs-troponin is statistically significant as a biomarker for acute myocardial infarction but not without using additional clinical risk stratification.

The presence of higher percentage of diabetes, which is a known risk factor for atherosclerotic cardiovascular events, as well as a higher median Hs-Troponin value in group A patients representing a higher chance of acute coronary syndrome and the likelihood of an underlying obstructive coronary artery disease as the myocardial damage is greater. The absence of significant difference between the groups in the confounding variables such as smoking status, history of hypertension, LDL value, creatinine, hemoglobin and CPK; gives more weight to use Hs-troponin as a determinant factor for predicting obstructive coronary artery disease.

An alternative diagnoses were found in those with a non-obstructive CAD (group B). Cardiac diagnoses were found in $32 \%$ of them which included valvular diseases, arrhythmogenic cardiomyopathy, 
myopericarditis, myocarditis, chest trauma and arrythmias; bigeminy, ventricular tachycardia, and supraventricular tachycardia. This indicates that the elevated troponin in these patients was due to a myocardial injury rather than a myocardial ischemic infarction. Furthermore, $20 \%$ of group B had noncardiac diagnoses which included bladder cancer, sarcoidosis, H.Pylori infection, gastric ulcer, and anemia. Several published articles support that these alternative cardiac and non-cardiac diagnoses are associated with elevated hs-troponin without any significant obstructive coronary artery disease [14-18].

Although our sample size is calculated to confer $80 \%$ power, our research is uni-centered and conducted in one-year time interval; a limitation that can be easily overcome by further research. This will confer more power and clarify the accuracy of Hs-troponin in detection of obstructive coronary artery disease in patients admitted with NSTEMI.

\section{Conclusion}

Our study concludes that at the current cutoff point of $14 \mathrm{ng} / \mathrm{L}$ is less specific in detecting obstructive coronary artery disease. A new level of $127 \mathrm{ng} / \mathrm{L}$ is proposed as it holds a good specificity for detecting obstructive coronary artery disease. Further study is recommended to evaluate this result in a large-scale population. The additional use of clinical cardiac risk stratification can improve the accuracy of Hstroponin in predicting obstructive coronary artery disease.

\section{Declarations}

\section{Ethical Approval and consent to participate:}

An ethics approval was obtained from Dubai Scientific Research Ethics Committee, DHA holding the reference number of DSREC/RRP/2019/13.

\section{Availability of Data and material}

Data is available with the authors in an excel sheet.

\section{Consent for publication}

Not applicable

\section{Competing interests}

Authors have no relationships relevant to the contents of this paper to disclose.

\section{Funding}

No funding source was for this manuscript.

\section{Authors' Contributions}


All Authors have read and approved the manuscript

OA: Has did the data collection and statistic analysis.

AA Has formulated the methodology, did the data base of variable collection and written a part of the discussion.

$A B$ : Formulated the aim of the research and assured integrate of the scientific content. In addition, edited the final manuscript.

HA: Has help in the data collection and written a part of the discussion.

SB: Has provided the patients files that fulfil the inclusion criteria and edited the final manuscript.

\section{Acknowledgments:}

The authors would like to thank Dr. Marwan Zidan for statistic studies and the Cardiology Department of Rashid Hospital for their support.

\section{Abbreviations}

NSTEMI : Non-ST Elevation Myocardial Infarction

ACS: Acute Coronary Syndrome

Hs-troponin: High sensitivity troponin

CK-MB: Creatine Kinase-Myocardial Band

CAG: Coronary angiography

CAD: Coronary artery disease

AUC: Area Under the Curve

ROC: receiver operating characteristic curve

AHA: American Heart Association

ACC: American College of Cardiology

MINOCA: Myocardial Infarction/Injury with Non-obstructive Coronary Arteries

\section{References}


[1] Pustjens, T.F.S., Appelman, Y., Damman, P. et al. Guidelines for the management of myocardial infarction/injury with non-obstructive coronary arteries (MINOCA): a position paper from the Dutch ACS working group. Neth Heart J (2019). https://doi.org/10.1007/s12471-019-01344-6

[2] Collste $\mathrm{O}$, Sorensson P, Frick M, et al. Myocardial infarction with normal coronary arteries is common and associated with normal findings on cardiovascular magnetic resonance imaging: results from the Stockholm Myocardiallnfarction with Normal Coronaries study. J Intern Med.2013;273(2):189-96.

[3] Pasupathy S, Tavella R, Beltrame JF. Myocardial infarction with Nonobstructive coronary arteries (MINOCA):the past, present, and future management.Circulation.2017;135(16):1490-3

[4] Ezra A. Amsterdam, Nanette K. Wenger, Ralph G. Brindis, Donald E. Casey, Theodore G. Ganiats, David R. Holmes, Allan S. Jaffe, Hani Jneid, Rosemary F. Kelly, Michael C. Kontos, Glenn N. Levine, Philip R. Liebson, Debabrata Mukherjee, Eric D. Peterson, Marc S. Sabatine, Richard W. Smalling, Susan J. Zieman. J Am Coll Cardiol. 2014 Dec, 64 (24) e139-e228.

[5] Roe M T, Harrington R A, Prosper D M, etal. Clinical and therapeutic profile of patients presenting with acute coronarySyndromes who do not have significant coronary artery disease. The Platelet Glycoprotein Ilb/Illa in Unstable Angina:Receptor Suppression Using Integril in Therapy (PURSUIT) trial investigators. Circulation. 2000;102(10):1101-6

[6] Safdar B, Spatz ES, Dreyer RP, et al. Presentation, clinical profile, and prognosis of young patients with myocardial infarction with nonobstructive coronary arteries (MINOCA): results from the VIRGO study. J Am Heart Assoc. 2018; https://doi.org/10.1161/JAHA.118.009174.

[7] Larsen Al, Nilsen DW, Yu J, et al. Long-term prognosis of patients presenting with ST-segment elevation myocardial infarction with no significant coronary artery disease (from the HORIZONS-AMI trial). Am J Cardiol. 2013;111(5):643-8

[8] Patel MR, Chen AY, Peterson ED, et al. Prevalence, predictors, and outcomes of patients with non-STsegment elevation myocardial infarction and insignificant coronary artery disease: results from the Can Rapid risk stratification of Unstable angina patients Suppress Adverse outcomes with Early implementation of the ACC/AHA Guidelines (CRUSADE)initiative. AmHeartJ.2006;152(4):641-7.

[9] Vasile V., Jaffe A. High-Sensitivity Cardiac Troponin in the Evaluation of Possible AMI. American College of Cardiology. Expert Analysis. July 2018. Retrieved 24 ${ }^{\text {th }}$ September 2020.

[10] Collet J., Thiele H., Barbato E., et al. 2020 ESC Guidelines for the management of acute coronary syndromes in patients with persistent ST-segment elevation. European Heart Journal (2020) 00, 179. doi:10.1093/eurheartj/ehaa575

[11]Braunwald E, Antman EM, Beasley JW, et al. ACC/AHA 2002 guideline update for the management of patients with unstable angina and non-ST-segment elevation myocardial infarction - summary article: a 
report of the American College of Cardiology/American Heart Association task force on practice guidelines (Committee on the Management of Patients With Unstable Angina) J Am Coll Cardiol. 2002;40:1366-1374.

[12] Alpert JS, Thygesen K, Antman E, et al. Myocardial infarction redefined - a consensus document of The Joint European Society of Cardiology/American College of Cardiology Committee for the redefinition of myocardial infarction. J Am Coll Cardiol. 2000;36:959-969.

[13] Mandrekar J. Receiver operating characteristic curve in diagnostic test assessment. J Thorac Oncol. 2010; 5(9):1315-1316. doi: 10.1097/JT0.0b013e3181ec173d.

[14] Korff S., Katus H., Giannitsis E. Differential diagnosis of elevated troponins. Heart 2006; 92:987-993. doi: 10.1136/hrt.2005.071282

[15] Kanjwal, K., Imran, N., Grubb, B., \&Kanjwal, Y. (2008). Troponin elevation in patients with various tachycardias and normal epicardial coronaries. Indian pacing and electrophysiology journal, 8(3), 172174.

[16] Kelley W, Januzzi J, Christenson R. Increases of Cardiac Troponin in Conditions other than Acute Coronary Syndrome and Heart Failure. Clinical Chemistry. 2009; 55(12): 2098-2112.

[17] Ralli S, Horwich TB, Fonarow GC. Relationship between anemia, cardiac troponin I, and B-type natriuretic peptide levels and mortality in patients with advanced heart failure. Am Heart J. 2005;150(6):1220-1227.

[18] Kandolin R, Lehtonen J, Airaksinen J, Vihinen T, Miettinen H, Kaikkonen K, Haataja P, Kerola T, Kupari M. Usefulness of cardiac troponins as markers of early treatment response in cardiac sarcoidosis. Am $\mathrm{J}$ Cardiol2015;116:960-964.

\section{Tables}


Table.1 Comparison of different variables between CAG

\begin{tabular}{|c|c|c|c|}
\hline \multirow[t]{2}{*}{ Variables } & \multicolumn{2}{|l|}{ Group } & P-Value \\
\hline & \multicolumn{3}{|c|}{ CAG PositiveCAG Negative } \\
\hline Smoker (\%) & 37 & 40 & 0.799 \\
\hline Diabetic (\%) & 54 & 39 & 0.014 \\
\hline Hypertensive (\%) & 54 & 60 & 0.588 \\
\hline \multicolumn{2}{|c|}{ Median hs-Troponin (ng/L)145 } & 54 & 0.030 \\
\hline Median HBA1C (\%) & 6.100 & 5.750 & 0.135 \\
\hline Mean LDL (mg/dL) & 126.48 & 121.22 & 0.567 \\
\hline
\end{tabular}

Table.2 Comparing cofounding variables between patients with positive coronary angiography finding to patients with a negative coronary angiography finding.

\begin{tabular}{|lll|}
\hline Variable & \multicolumn{2}{c|}{ Group A (Positive CAG)Group B (Negative CAG) } \\
\hline Median Creatinine $(\mathrm{mg} / \mathrm{dL})$ & 0.9 & 0.8 \\
\hline Mean Hemoglobin $(\mathrm{g} / \mathrm{dL})$ & 14.25 & 14.21 \\
\hline Median CPK $(\mathrm{mg} / \mathrm{L})$ & 216 & 191 \\
\hline
\end{tabular}




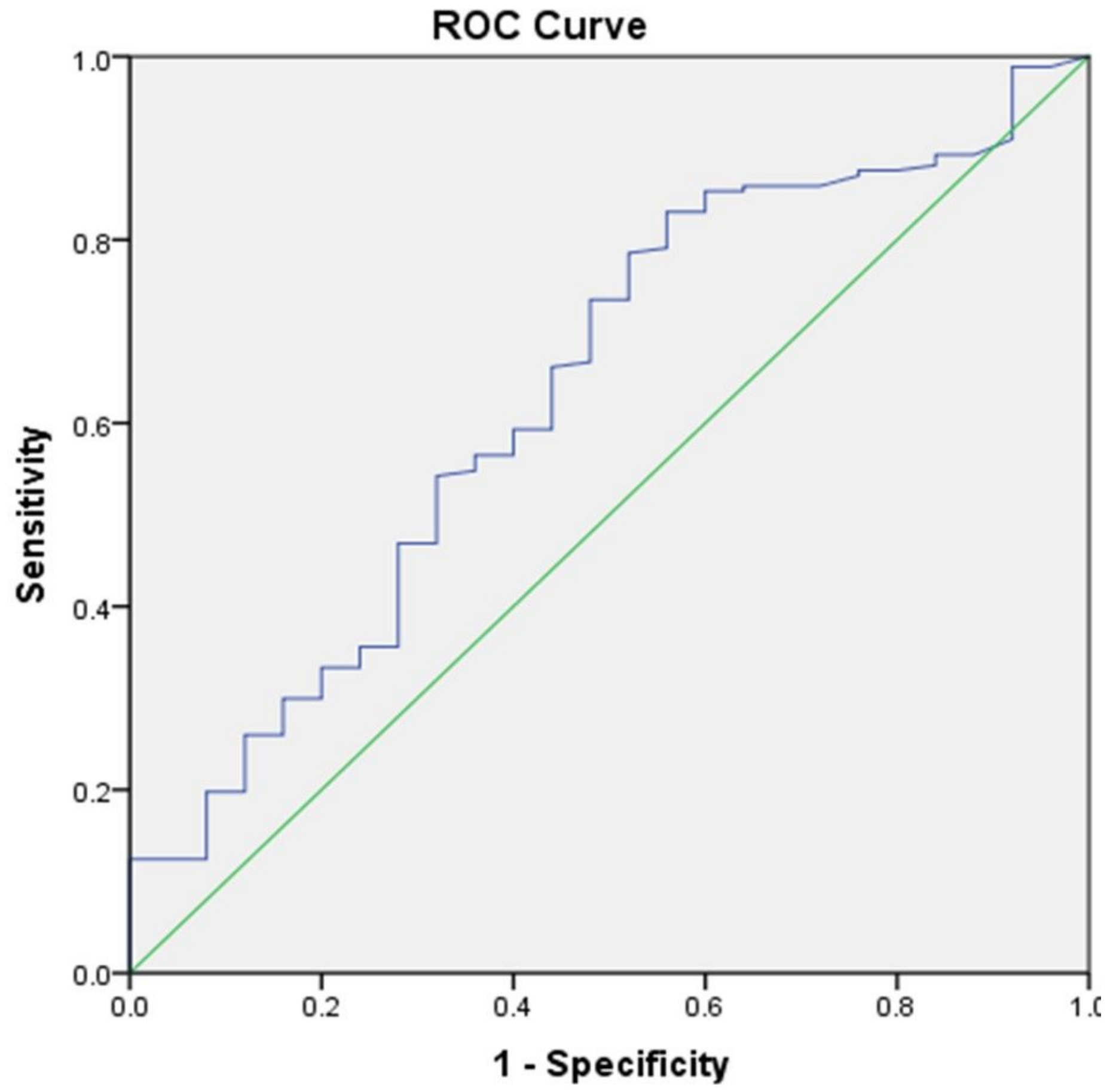

Diagonal segments are produced by ties.

Figure 1

ROC Curve of our data

\section{Supplementary Files}

This is a list of supplementary files associated with this preprint. Click to download. 
- Questionnaire.xIsx

Page $11 / 11$ 